\title{
A Noninvasive Quantum Thermometer
}

\section{A quantum dot can measure ultracold temperatures without the need for direct electrical connections to the outside world.}

\section{By Christopher Crockett}

A ccurate measurements of temperature are essential for understanding the behavior of ultracold circuits, such as the ones used in quantum computers. While a number of "electron thermometers" exist for this purpose, they all require a direct electrical connection to the circuit, something that can interfere with the circuit's electronic behavior. Now, researchers have circumvented that requirement with a quantum-dot thermometer that can accurately measure temperatures down to $1 \mathrm{~K}$ without a direct electrical connection [1].

As a circuit's temperature changes, so does the range of energies that electrons can occupy in the circuit. This distribution is observable in a component of a quantum dot's capacitance known as the "tunneling capacitance." Previous quantum dot thermometers worked by measuring the current through the dot or by direct charge sensing. Joshua Chawner of Lancaster University in the UK and colleagues instead use an indirect approach that leverages this tunneling capacitance.

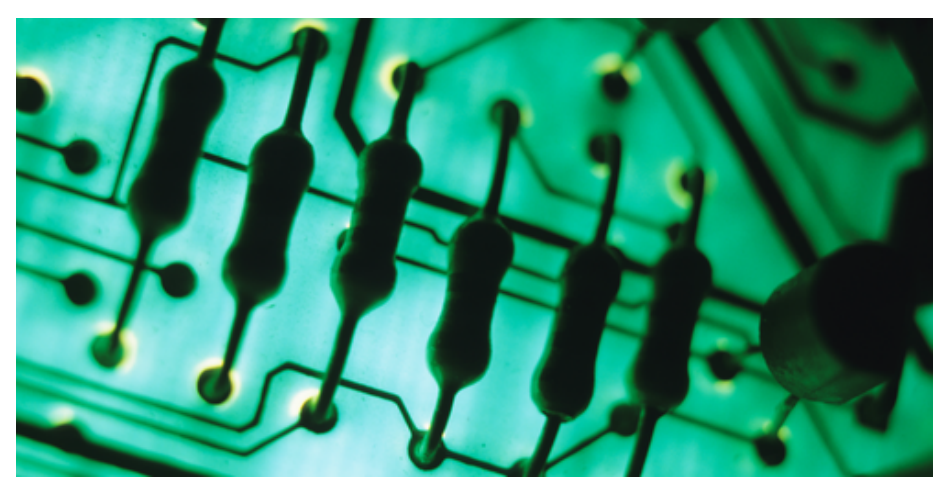

Credit: Mario Beauregard/stock.adobe.com
In their experiments, the quantum dot was close to, but not directly contacting, one gate of a transistor in an electric circuit. A radio-frequency signal was sent through the circuit to this gate. They then measured the returned signal's phase, which shifted as the dots' tunneling capacitance changed.

Testing the thermometer in a cryogenic refrigerator-with a second thermometer as a check-the team demonstrated that their setup works for temperatures down to about $1 \mathrm{~K}$. Below that, the readouts from the two thermometers diverged but stayed within one standard deviation of each other. The team is now working on improving the quantum dot thermometer's accuracy below $1 \mathrm{~K}$, which they say will require redesigning the quantum dot to alter its tunneling capacitance.

Christopher Crockett is a freelance writer based in Arlington, Virginia.

\section{REFERENCES}

1. J. Chawner et al., "Nongalvanic calibration and operation of a quantum dot thermometer," Phys. Rev. Applied 15, 034044 (2021). 